\title{
Combination of icotinib, surgery, and internal- radiotherapy of a patient with lung cancer severely metastasized to the vertebrae bones with EGFR mutation: a case report
}

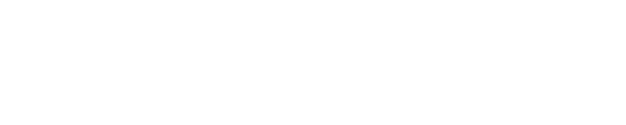

\section{Li-Li Qu \\ Hai-Feng Qin \\ Hong-Jun Gao \\ Xiao-Qing Liu}

Department of Lung Cancer, Affiliated Hospital of Academy of Military Medical Science, Beijing, People's Republic of China
Correspondence: Xiao-Qing Liu Department of Lung Cancer, Affiliated Hospital of Academy of Military Medical Science, No 8 East Road, Fengtai District, Beijing 10007I, People's Republic of China

Tel +861066947163

Fax +86 I0 5III28605

Email liuxq@medmail.com.cn

\begin{abstract}
A 48-year-old Chinese female was referred to us regarding EGFR-mutated advanced non-small cell lung cancer, and metastasis to left scapula and vertebrae bones which caused pathological fracture at T8 and T10 thoracic vertebrae. An aggressive combined therapy with icotinib, vertebrae operation, and radioactive particle implantation and immunotherapy was proposed to prevent paraplegia, relieve pain, and control the overall and local tumor lesions. No postoperative symptoms were seen after surgery, and the pain was significantly relieved. Icotinib merited a 31-month partial response with grade 1 diarrhea as its drug-related adverse event. High dose of icotinib was administered after pelvis lesion progression for 3 months with good tolerance. Combination therapy of icotinib, surgery, and internal radiation for metastases of the vertebrae bones from non-small cell lung cancer seems to be a very promising technique both for sufficient pain relief and for local control of the tumor, vertebrae operation can be an encouraging option for patients with EFGR positive mutation and good prognosis indicator.
\end{abstract}

Keywords: lung cancer, spinal metastasis, pathological fracture, spinal canal stenosis, icotinib

\section{Introduction}

The vertebrae bones are the most commonly metastasized sites from advanced non-small cell lung cancer (NSCLC) with poor prognosis, especially for those with spinal cord compression and pathologic fracture. Currently, there is still no standard therapy guideline for those patients. The choice of surgery is questionable due to high surgical morbidity and limited survival benefit. ${ }^{1}$ Therefore, it is essential to identify patients with better prognosis factors, such as positive EGFR gene mutation, good performance status (PS), and nonsmoking status. Icotinib is an orally administered small-molecule reversible EGFR tyrosine kinase inhibitor (EGFR-TKI) with favorable antitumor activities and safety profile. ${ }^{1}$ Here we present a patient with long progression-free survival (PFS) after aggressive combined first-line therapy including icotinib, surgery, and inner-radiotherapy.

\section{Case report}

A 48-year-old Chinese female non-smoker presented at our hospital with a 4-month history of left scapula and back pain (visual analog scale score: 6/10). She was found to have stage IV adenocarcinoma (T2N2M1, Figures 1 and 2) with multiple bone and brain metastases. Computed tomography (CT) scan revealed an osteolytic lesion $11 \mathrm{~cm}$ in size in the left scapula, magnetic resonance imaging (MRI) revealed pathological 

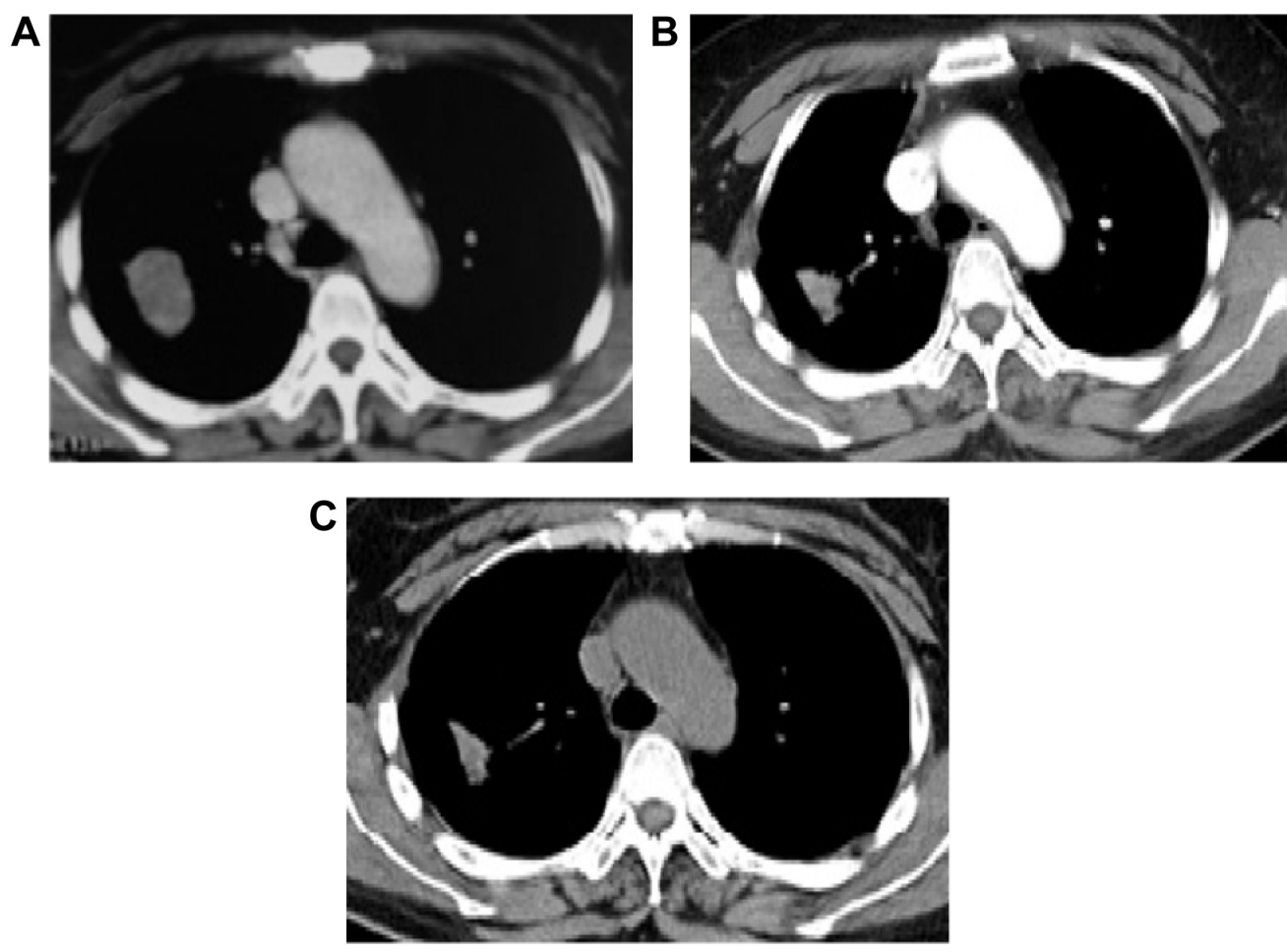

Figure I The computed tomography scan at diagnosis with a $3.9 \mathrm{~cm}$ bean-shaped tumor in the upper lobe of right lung (A). The lesion had significant reduction in tumor size I month later (B), and remained stable for 31 months with oral icotinib therapy (C).

fracture and spinal canal stenosis at T8 and T10 thoracic vertebrae (Figure 3), and small nodules on the cerebellum and bilateral temporal lobe (Figure 4). Her PS score was 2. Mutation analysis by amplification refractory mutation system showed that the tumor had positive EGFR mutation of exon 19 deletion.

Given the fracture, the high risk of paraplegia due to spinal cord compression, good bodily functions with no medical comorbidities, and the positive EGFR mutation, an internal fixation between $\mathrm{T} 7$ and $\mathrm{T} 11$ via retroperitoneal approach

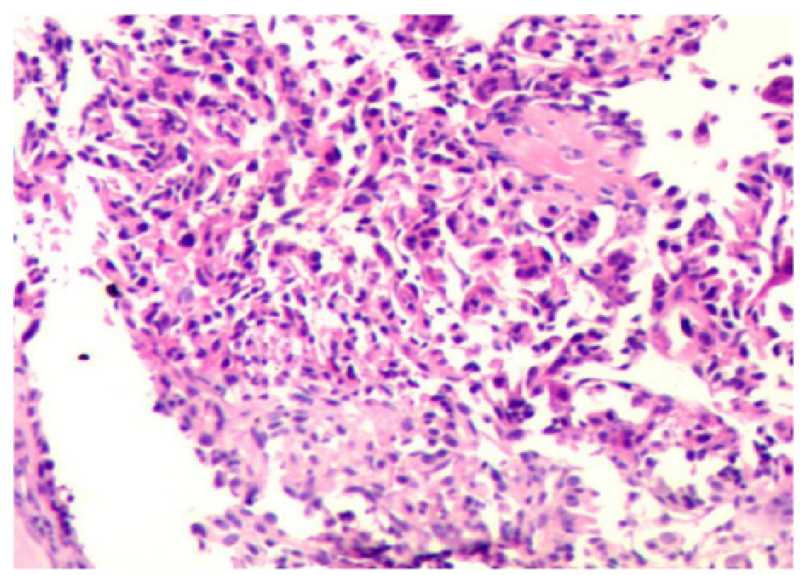

Figure 2 Stained sample of bone metastasis of lung adenocarcinoma, original magnification $\times 400$. combined with an implantation of radioactive particle into T8 and T10 spine (Figure 3 C,D) was performed. Additional cement packing was also performed during surgery to improve the stability. Immediately after the surgery, icotinib was orally administered (125 mg, three times per day) as firstline treatment. The primary icotinib-related adverse event (AE) was grade 1 diarrhea which resolved in 1 week without any medication (according to common terminology criteria for AE v4.0, CTCAE). In addition, autologous DC-CIK cells amplified in vitro were given by venous reinfusion once every two days after surgery to stimulate immunologic function as a supplementary therapy. With respect to the brain metastasis, whole brain irradiation therapy was not recommended since the intracranial lesions were all small scattered nodules, and no neurological symptoms were seen.

A partial response (PR) was seen after 1 month icotinib treatment (Figure $1 \mathrm{~B}$ ), and this PR persisted for 31 months (November 16, 2014) during which the Eastern Cooperative Oncology Group PS of the patient remained 1. The left scapula and back pain was significantly relieved (visual analog scale score 0 ) in 1 month without any surgery-related complication or AEs. Moreover, the plasma CEA level also fell to the normal level and was maintained.

However, 31 months after the procedure (November 16, 2014), the patient developed pelvic pain. The ECT scans 

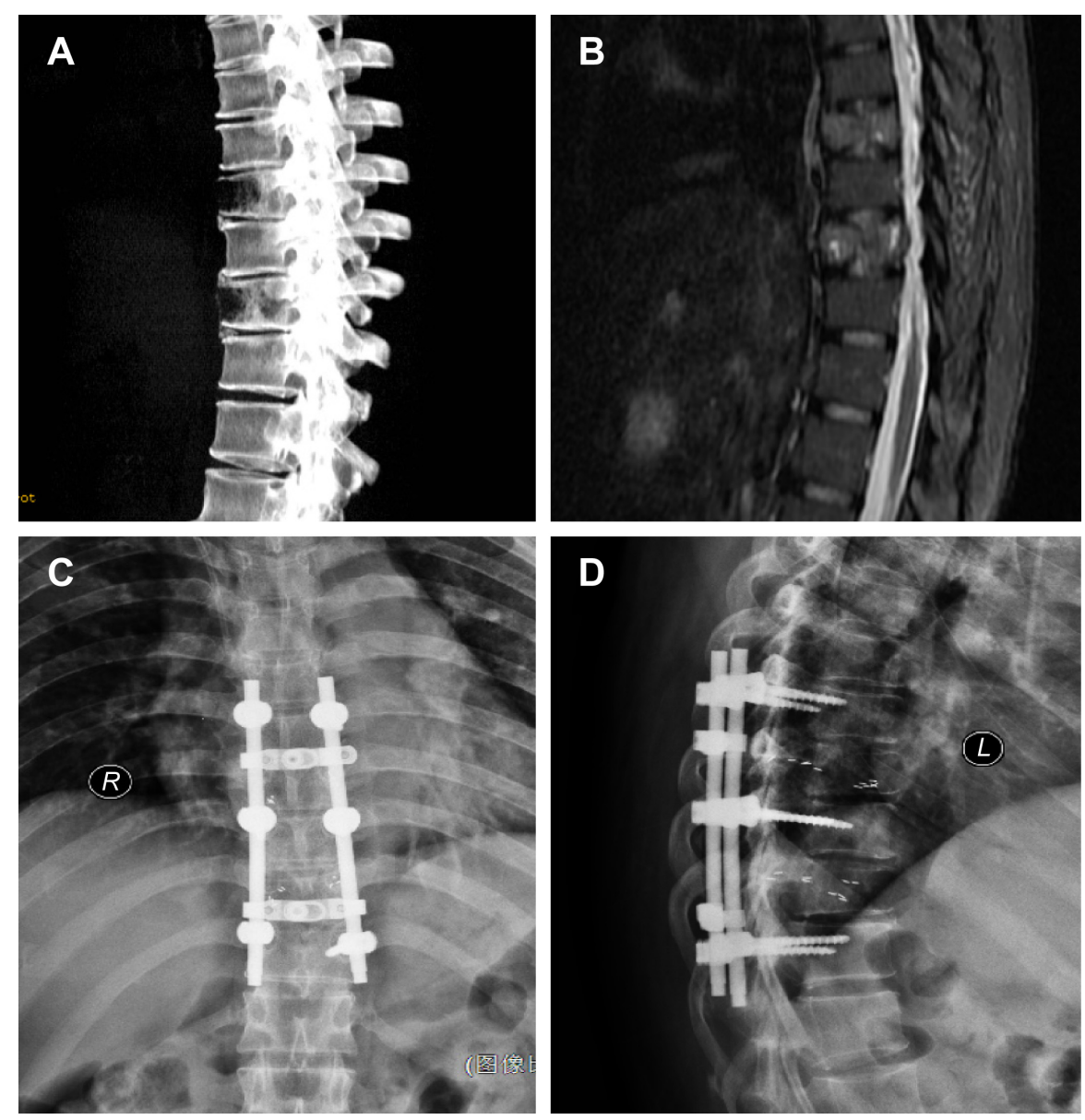

Figure 3 Computed tomography (CT) and magnetic resonance imaging scan of spine. Notes: T8 and TI0 pathological fracture with local spinal canal stenosis (A, B). CT scan after surgery via retroperitoneal approach and radioactive particle implantation into T8 and TI 0 spine (C, D).

revealed higher radioactive concentration on right iliac bone (Figure 5) without other progression according to systematic examination. Radiotherapy was then administered to the iliac bone lesions with a total dose of $60 \mathrm{~Gy} / 30$ fractions, 2 Gy per fraction. Meanwhile, $250 \mathrm{mg}$ icotinib was given, and no drug-related AEs were seen. The local pain was improved after radiotherapy and higher dose icotinib therapy.

\section{Discussion}

Lung cancer is one of the most common cancers and continues to be the leading cause of cancer-related deaths worldwide. With high malignancy, 30\%-40\% patients with advanced lung cancer may have bone metastasis at diagnosis, with the most common sites at vertebrae and proximal bone of the trunk. ${ }^{2}$ The prognosis of patients with skeletalrelated events (SRE) is usually poor, especially those with serious SRE including pathological fracture and spinal cord compression. ${ }^{3}$ Sugiura et al reported that the 6-month, 1-year, and 2-year survival rates of patients afflicted with lung cancer metastasized to the vertebrae bones were $59.9 \%, 31.6 \%$, and only $11.3 \%$, respectively. ${ }^{4}$ The management and treatment for those patients is challenging. Multidisciplinary approaches including surgery, radiotherapy, chemotherapy, and targeted therapy are needed, which require identification of patients to reduce risks from aggressive therapies while achieving maximum clinical benefits.

In this case, we performed surgical treatment, internal radiation by radioactive particle implantation, followed with target therapy and immunotherapy. Significant benefit was seen from the aggressive combined therapy, and the patient had 31 months of PR without tumor-related symptoms. This case demonstrates that surgical intervention can be considered for certain patients with lung cancer metastasized to the vertebrae bones; however, deliberations are needed to identify potential patients.

According to the NCCN guidelines, first-line EGFR-TKIs is the standard therapy for treatment-naïve, advanced NSCLC patients harboring positive EGFR mutation. ${ }^{5}$ The efficacy of 

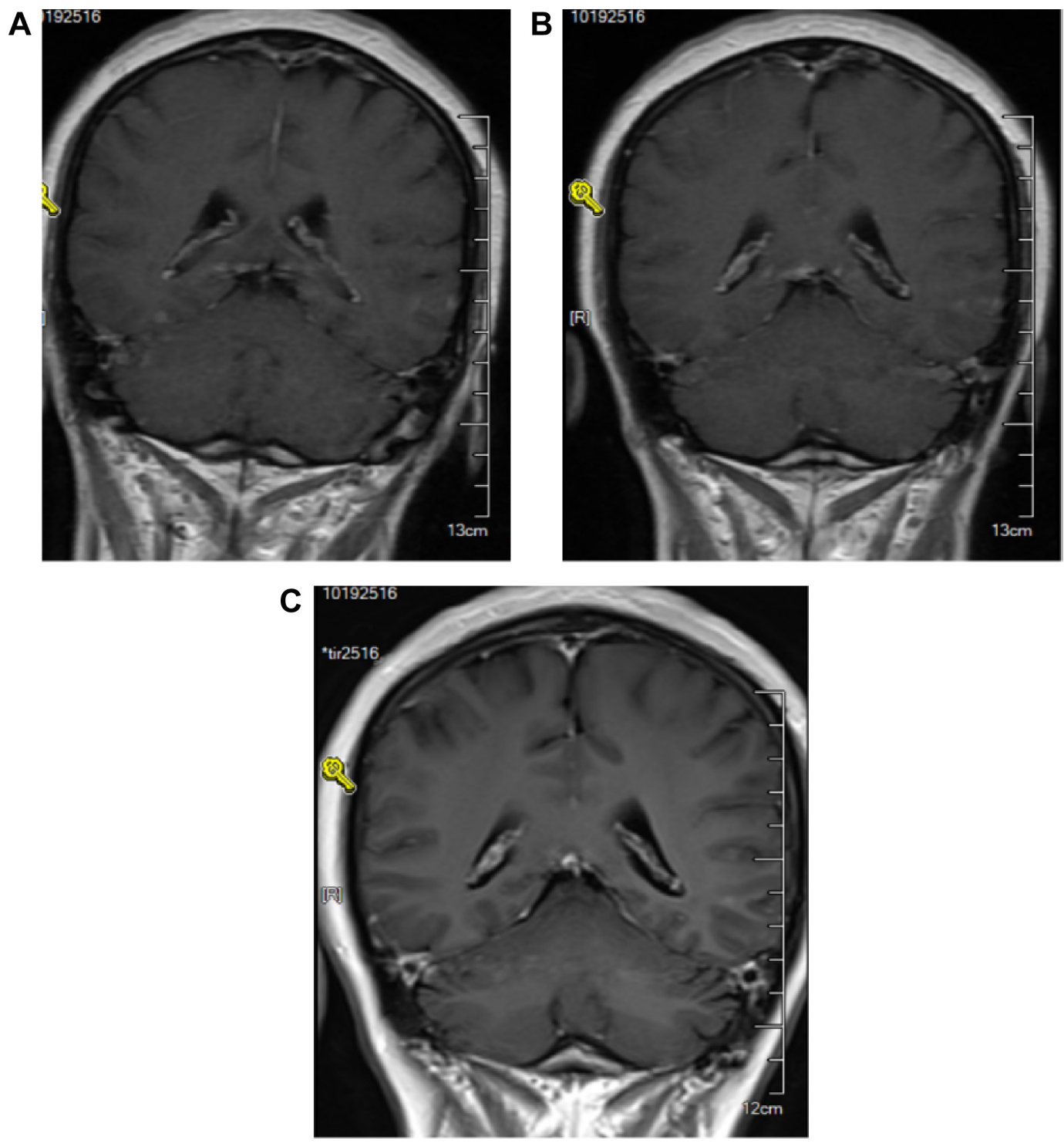

Figure $4 \mathrm{MRI}$ scan of brain (scattered small nodules on the cerebellum and bilateral temporal lobe).

Notes: The lesions remained stable at diagnosis (A), I month (B), and 31 months later (C). R=right side.

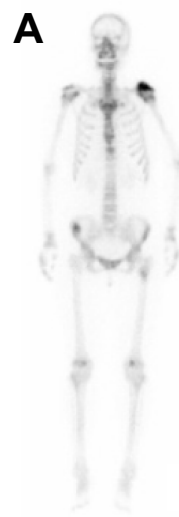

Anterior

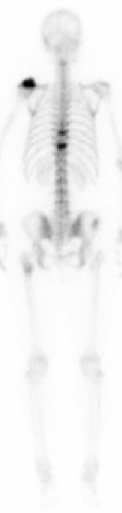

Posterior

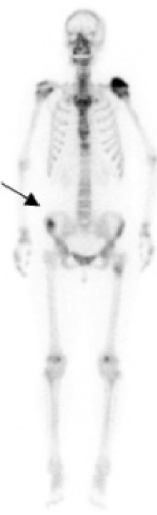

Anterior

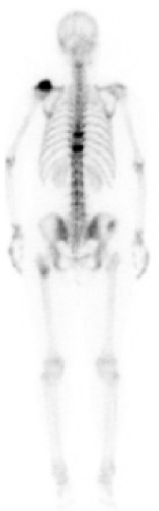

Posterior
B

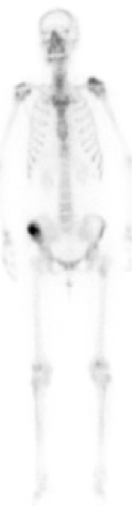

Anterior

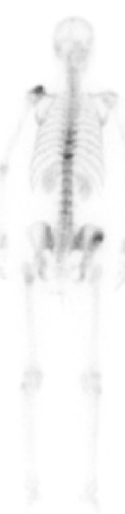

Posterior

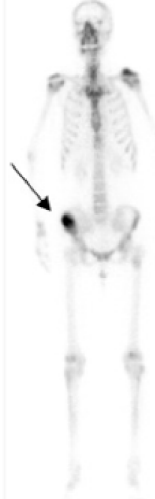

Anterior

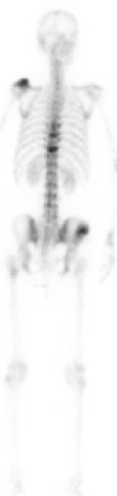

Posterior

Figure 5 ECT scan at diagnosis (A) and after 31 months icotinib treatment (B).

Note: The arrow shows higher radioactive concentration on right iliac bone in $B$ than $A$.

Abbreviation: ECT, electrical capacitance tomography. 
EGFR-TKIs has been proven in many large-scale randomized Phase III trials, especially for Asian, female, never-smoker, and adenocarcinoma patients. ${ }^{6}$ In addition, NSCLC patients with positive $E G F R$ mutation can further benefit from EGFR inhibitors, especially, exon 19 deletions were reported to be associated with longer PFS compared with $L 858 R$ mutation at exon 21 of EGFR. ${ }^{7,8}$ Icotinib is an orally administered small molecular EGFR-TKI autonomously researched and developed by the Chinese. The chemical structure of icotinib is similar to other EGFR-TKIs such as erlotinib and gefitinib, while icotinib has a shorter half-time of 6-8 hours and has to be taken three times a day. ${ }^{9}$ Icotinib is an oral selective EGFRTKI which was approved for treating advanced NSCLC patients who failed with previous chemotherapies by China Food and Drug administration in June 2011. Its approval was based on the registered Phase III trial (ICOGEN) which showed that icotinib is non-inferior to gefitinib in terms of PFS, ORR, DCR, and overall survival in both unselected and EGFR mutated NSCLC patients who failed with previous chemotherapies. The PFS for icotinib and gefitinib was 4.6 and 3.4 months (hazard ratio $0.84 ; P=13$ ), respectively. Furthermore, icotinib was associated with lower incidence of drug-related AEs (61\% vs 70\%, $P=0.046)$ and diarrhea $(19 \%$ vs $28 \%, P=0.033) .{ }^{10}$ Meanwhile, high doses of icotinib may overcome the resistance to the standard dose of EGFR-TKI. It is reported that icotinib is well-tolerated at doses of 75-250 mg three times a day. ${ }^{11}$

And as to the NCCN guidelines, patients with symptomatic bone metastasis and high risk of pathological fracture are recommended to receive surgery and external radiotherapy. Surgery can provide mechanical stabilization, pain relief, and maintenance of neurological function; however, it is reported that less than $10 \%$ of patients with spinal metastasis undergo surgery. ${ }^{12,13}$ Therefore, surgical risks must be weighed against life expectancy and quality of life (QoL) for surgical intervention. With respect to radiotherapy, patients with radiosensitive tumors, with expected survival of less than 3 months, inability to tolerate an operation, total neurological deficit for more than 24-48 hours, and multi-level or diffuse disease are fit for palliative external radiation therapy. ${ }^{14}$

In our case, considering the remarkable response to icotinib in the bone metastasis lesions, and prevention of decreased QoL due to repeated positioning and long-term treatment during the external radiation therapy, we chose internal radiation instead. This case revealed the feasibility of aggressive combined therapy: surgery reduced the risk of paralysis due to spinal pathological fracture, and the internal radiation by radioactive particle implantation enhanced local control of the spinal lesion, which lead to improved QoL and less SREs.

Adoptive cell immunotherapy is a novel approach that relies on an ex vivo expansion of the autologous tumorspecific effector cells before reinfusion into the host. ${ }^{15,16}$ Immunological effector cells such as DC and CIK cells have been employed to treat cancer and eliminate residual tumor cells after surgery. In this case, the patient had DC and CIK cells collection before any tumor-related therapy. Palliative vertebrae surgery may disrupt the micro environment of the immune system and expanded DC-CIK cells with high cell immunological activity may help with rebuilding of a balanced immune system. A recent study revealed that patients with erlotinib plus DC and CIK cell therapy had improved levels of immunological markers compared to erlotinib therapy alone. ${ }^{17}$ Our patient showed a good response and tolerance with the EGFR-TKI and cell immunological therapy.

Additionally, the patient had the advantages of relatively young age, good PS, few physical complications, no paraplegia, and positive EGFR mutation of exon 19 deletion. It should also be noted that this result is limited to our case report; it is of vital importance to have comprehensive evaluation for NSCLC patients with spinal metastasis.

\section{Conclusion}

In conclusion, the combination of surgery, internal radiation, EGFR-TKIs, and immunotherapy was effective and well-tolerated in terms of pain relief and local control of the tumor. However, clinicians should carefully consider the risk of aggressive therapy. Further experience is needed to assess the feasibility, efficacy, and safety of this combined therapy.

\section{Acknowledgment}

Supported by Capital Medical Development (No 2007-3042) and the National Key Foundation for Exploring Scientific Instrument (No 2011YQ170067).

\section{Disclosure}

The authors declare that they have no financial or other conflicts of interest in relation to this research and its publication.

\section{References}

1. Hu S, Xie G, Zhang DX, et al. Synthesis and biological evaluation of crown ether fused quinazoline analogues as potent EGFR inhibitors. Bioorg Med Chem Lett. 2014;22(19):6301-6305.

2. Delea T, Langer C, Mckiernan J, et al. The cost of treatment of skeletalrelated events in patients with bone metastases from lung cancer. Oncology. 2004;67(5-6):390-396. 
3. Lorusso V, Duran I, Garzon-Rodriquez C, et al. Health resource utilization associated with skeletal-related events in European patients with lung cancer: A subgroup analysis from a prospective multinational study. $\mathrm{Mol}$ Clin Oncol. 2014;2(5):701-708.

4. Sugiura H, Yamada K, Sugiura T, Hida T, Mitsudomi T. Predictors of survival in patients with bone metastasis of lung cancer. Clin Orthop Relat Res. 2008;466(3):729-736.

5. NCCN Clinical Practice Guidelines in Oncology (NCCN Guidelines) (Version 3. 2014). http://www.nccn.org/professionals/physician_gls/ pdf/nscl.pdf. Date:2014.04.17.

6. Wang J, Nong J, Jia H, et al. Efficacy and predictors of EGFR tyrosine kinase inhibitors in Chinese advanced lung adenocarcinoma: analyses of 253 cases from a single institute. Oncol Res. 2014;21(5):237-246.

7. Paez JG, Janne A, Lee JC, et al. EGFR mutations in lung cancer correlation with clinical response to gefitinib therapy. Science. 2004; 304(5676):1497-1500.

8. Wenhua Liang, Jin Sheng, Xuan Wu, et al. Exon 19 deletion association with progression-free survival compared to L858R mutation at exon 21 in treatment with first-line EGFR-TKIs: A meta-analysis of subgroup data from eight phase III randomized controlled trials. 2014 ASCO Annual Meeting Abstracts. Journal of Clinical Oncology. 2014;32 (15 Suppl):8107.

9. Zhao Q, Shentu J, Xu N, et al. Phase I study of icotinib hydrochloride (BPI-2009H), an oral EGFR tyrosine kinase inhibitor in patients with advanced NSCLC and other solid tumors. Lung Cancer. 2011;73(2): 195-202.
10. Shi Y, Zhang L, Liu X, et al. Icotinib versus gefitinib in previously treated advanced non-small-cell lung cancer (ICOGEN): a randomised, double-blind phase 3 non-inferiority trial. Lancet Oncol. 2013;14(10):953-961.

11. Guan Y, Zhao H, Meng J, Yan X, Jiao S. Dramatic response to highdose icotinib in a lung adenocarcinoma patient after erlotinib failure. Lung Cancer. 2014;83(2):305-307.

12. Simmons ED, Zheng Y. Vertebral tumors: surgical versus nonsurgical treatment. Clin Orthop Relat Res. 2006;443:233-247.

13. Bartels RH, van der Linden YM, van der Graaf WT. Spinal extradural metastasis: review of current treatment options. CA Cancer J Clin. 2008;58(4):245-259.

14. Bhatt AD, Shuler JC, Boakye M, Woo SY. Current and emerging concepts in non-invasive and minimally invasive management of spine metastasis. Cancer Treat Rev. 2013;39(2):142-152.

15. Choi D, Kim TG, Sung YC. The past, present, and future of adoptive I cell therapy. Immune Netw. 2012;12(4):139-147.

16. Sangiolo D. Cytokine induced killer cells as promising immunotherapy for solid tumors. J Cancer. 2011;2:363-368.

17. Shi SB, Tang XY, Tian J, et al. Efficacy of erlotinib plus dendritic cells and cytokine-induced killer cells in maintenance therapy of advanced non-small cell lung cancer. J Immunother. 2014;37(4):250-255.
OncoTargets and Therapy

\section{Publish your work in this journal}

OncoTargets and Therapy is an international, peer-reviewed, open access journal focusing on the pathological basis of all cancers, potential targets for therapy and treatment protocols employed to improve the management of cancer patients. The journal also focuses on the impact of management programs and new therapeutic agents and protocols on

\section{Dovepress}

patient perspectives such as quality of life, adherence and satisfaction The manuscript management system is completely online and includes a very quick and fair peer-review system, which is all easy to use. Visit http://www.dovepress.com/testimonials.php to read real quotes from published authors. 